\title{
Sobre las recomendaciones del Ministerio de Salud para el tratamiento farmacológico de la COVID-19 en el Perú
}

\section{Regarding the MINSA recommendations on the pharmacological treatment of COVID-19 in Peru}

Correspondencia Germán Málaga Rodríguez german.malaga@upch.pe

Recibido: $21 / 06 / 2020$ Aprobado: 25/06/2020

Citar como: Chirinos JA Corrales-Medina VF, HeresiDávila $G$, Hernandez AV, Málaga $G$, Mallea JM, et al. Sobre las recomendaciones del Ministerio de Salud para el tratamiento farmacológico de la COVID-19 en el Perú. Acta Med Peru. 2020;37(2): 231-5. doi: https://doi.org/ 10.35663/amp.2020.372.1030
Julio A. Chirinos ${ }^{1, a, b}$, Vicente F. Corrales-Medina ${ }^{2, a, c}$, Gustavo Heresi-Dávila ${ }^{3, a, d}$, Adrian V. Hernandez ${ }^{4, e}$, Germán Málaga ${ }^{5, a}$, Jorge M. Mallea ${ }^{6, d}$, J. Jaime Miranda ${ }^{7,8}$, Oscar O. Morey9,a,f , Juan E. Rodríguez-Mori ${ }^{10,9}$, Jesús E. SalinasGamero ${ }^{11, d}$, José Serpa-Alvarez ${ }^{12, a, c}$, Alvaro Taype-Rondan ${ }^{13, e}$, Carol Zavaleta ${ }^{14}$

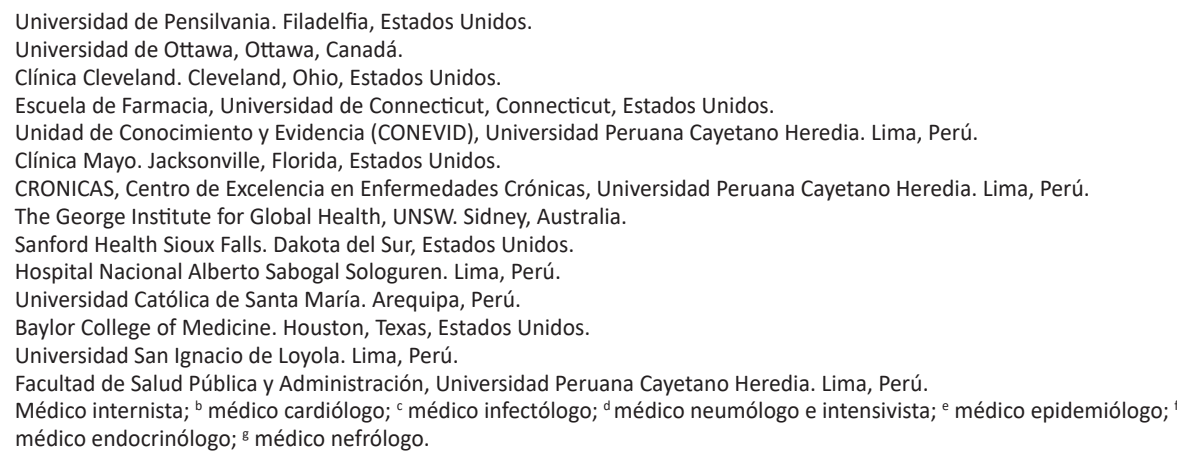

Sr. Editor,

Hemos seguido con atención la evolución de la COVID-19 en el Perú, las recomendaciones de tratamiento de la enfermedad por el comité de expertos del Ministerio de Salud (MINSA), y las declaraciones públicas sobre el tema de varios expertos. Esta carta deriva de nuestra opinión profesional y no constituye la posición oficial de nuestras instituciones clínicas o académicas.

Dada la crisis de salud pública que trajo consigo la COVID-19, el MINSA recomendó tempranamente el uso de hidroxicloroquina e ivermectina ${ }^{[1,2]}$. Después de que las recomendaciones fueron formuladas, en el caso de la hidroxicloroquina, se han concluido estudios importantes que reportan la falta de efectividad de este medicamento para tratamiento y prevención de la COVID-19 ${ }^{[3,4]}$ y que por el contrario, sugieren un riesgo de toxicidad en pacientes con esta infección, particularmente toxicidad cardíaca, sobre todo cuando se utiliza simultáneamente con azitromicina (un antibiótico que también es recomendado en los protocolos del MINSA para el tratamiento de la COVID-19) ${ }^{[5-8]}$. Basados en la evidencia de falta de eficacia, y en su riesgo de toxicidad, creemos que la recomendación de utilizar hidroxicloroquina en la COVID-19 debe ser reevaluada y descontinuada lo antes posible.

En el caso de la ivermectina, también ha quedado claro que los escasos estudios disponibles en los que se basa el entusiasmo por el uso de este medicamento para tratar la COVID-19 tienen severas limitaciones ${ }^{\left[{ }^{9}\right.}$. La ivermectina, un medicamento comúnmente utilizado en el tratamiento de enfermedades causadas por parásitos, ha demostrado tener un efecto contra el virus que causa la COVID-19 en experimentos de laboratorio utilizando cultivos de células de mono ${ }^{[10]}$. Sin embargo, las concentraciones necesarias para lograr un efecto antiviral en estos experimentos fueron 
aproximadamente 35 a 100 veces mayores que las que se alcanzan en humanos con las dosis actualmente recomendadas para uso médico. Es decir, es muy improbable que en pacientes con la COVID-19 que reciben las dosis que actualmente se consideran seguras en humanos, se produzca el efecto antiviral observado en los experimentos con células de mono. Por otro lado, aunque es cierto que las dosis de ivermectina que actualmente se usan en humanos son consideradas seguras (es decir, de bajo riesgo de efectos adversos severos), es preciso señalar que esta presunción proviene del uso de ivermectina en pacientes que no llegan al nivel de compromiso agudo que se observa en pacientes con esta infección, y que usualmente no tienen otras enfermedades crónicas preexistentes (del corazón, pulmones, riñones, hígado, sangre, etc.) que son más comunes en pacientes con la COVID-19.

Por otro lado, se debe tener en cuenta que se desconocen las posibles interacciones entre la ivermectina y múltiples medicamentos que se usan en pacientes con la COVID-19, sobre todo en aquellos que tienen enfermedades crónicas o están en la unidad de cuidados intensivos. Por estos motivos, creemos que el uso clínico de la ivermectina para el tratamiento de la COVID-19, como actualmente se recomienda, no está justificado y tiene un perfil de riesgo-beneficio muy incierto. Basados en una evaluación cuidadosa de la evidencia disponible, creemos que se debe descontinuar la recomendación del uso rutinario de ivermectina para tratar o prevenir la COVID-19, y restringir su uso a estudios de investigación debidamente justificados, controlados, monitorizados y regulados. Al mismo tiempo, no podemos dejar de reconocer la autonomía de los médicos peruanos para considerar el uso compasivo de este medicamento y por eso también se debe establecer un sistema de vigilancia de efectos adversos para aquellos pacientes con la COVID-19 que aún reciban ivermectina.

Es necesario reconocer que el uso de medicamentos con una pobre o desfavorable evidencia de eficacia en la COVID-19 como la hidroxicloroquina y la ivermectina, no solo tiene riesgos de potenciales efectos adversos, sino que también trae consigo un costo económico y un potencial costo de oportunidad para la implementación de otras intervenciones con eficacia establecidas como: lavado de manos, distanciamiento social y uso de mascarillas, pues las personas asumen un efecto protector e incluso profiláctico de la ivermectina, recomendación que ni siquiera ha sido evaluada.. La nota de prensa del ensayo controlado aleatorizado RECOVERY ${ }^{[11]}$ describió que dexametasona oral o intravenosa, una intervención de bajo costo y ampliamente disponible, trajo consigo un efecto sustancial de reducción de la mortalidad en pacientes hospitalizados con COVID-19 que requirieron ventilación mecánica o soporte con oxígeno, aunque aún se requiere evaluar los resultados completos de este estudio para tener una opinión más informada. Con relación al remdesivir, resultados de ensayos controlados aleatorizados, uno completo ${ }^{[12]}$ y uno preliminar ${ }^{[13]}$, en pacientes con la COVID-19 hospitalizados con neumonía e insuficiencia respiratoria indican que remdesivir intravenoso acortó la enfermedad (reducción en el tiempo a la recuperación hospitalaria vs. placebo, o el tiempo al que los pacientes ya no requerían oxígeno o cuidado médico continuo con cinco días de tratamiento vs. diez días de tratamiento). Un tercer estudio aleatorio más pequeño no demostró mejoría clínica (acortamiento de la enfermedad) estadísticamente significativa ${ }^{[14]}$. Un cuarto ensayo controlado aleatorizado ${ }^{[15]}$ ha sido reportado sólo como nota de prensa y falta la publicación final. Hasta el momento, no se ha demostrado que remdesivir disminuya la mortalidad de pacientes con COVID-19. Enfatizamos el alto costo del remdesivir comparado con la dexametasona.

En la Tabla 1 presentamos un contrapunto a argumentos comúnmente utilizados en favor del uso de hidroxicloroquina e ivermectina en el tratamiento de la COVID-19. En el Material suplementario, Apéndice 1 resumimos algunos conceptos útiles para el lector general que desea evaluar la evidencia a favor o en contra de estos medicamentos; en el Apéndice 2 declaramos nuestros conflictos de interés.

La respuesta del Gobierno del Perú a la COVID-19 ha tenido muchos aciertos que merecen amplio reconocimiento, en especial cuando se toman en cuenta los múltiples retos que implica una respuesta coordinada a esta pandemia. Sin embargo, enfocándonos solamente en el manejo clínico de la COVID-19, creemos que las recomendaciones ministeriales actuales para el tratamiento de esta enfermedad deben modificarse urgentemente, y se debe establecer una evaluación más rigurosa y dinámica para su tratamiento en el Perú, para beneficio de nuestra población. En este contexto, creemos que la hidroxicloroquina e ivermectina deben ser descontinuadas de los actuales protocolos clínicos para la COVID-19 y se debe enfatizar el uso de otros medicamentos con beneficio comprobado. Esperamos contribuir positivamente a la discusión de este asunto tan importante para nuestro país, sin ánimo de disminuir el mérito de las autoridades de salud, expertos del MINSA y los miles de colegas que con gran esfuerzo y dedicación tratan a nuestros enfermos en los varios centros de salud del Perú.

Para concluir, consideramos que es importante reconocer que las modificaciones de recomendaciones médicas en el contexto de una pandemia de tan rápido curso como la actual son perfectamente aceptables y no deben tener una connotación negativa, ya sea política o médica. Finalmente, creemos que el Perú se encuentra, por la capacidad de coordinación de políticas para el tratamiento de la COVID-19 en sus redes hospitalarias, en posición de ejecutar, de manera rápida, ensayos clínicos de alta calidad y gran escala para evaluar nuevas potenciales terapias para la COVID-19 de modo que se pueda contribuir al avance del conocimiento en la lucha mundial contra esta pandemia.

Contribuciones de autoría: según lo descrito en el Material Suplementario, Apéndice 2.

Potenciales conflictos de interés: los autores declaran no tener conflictos de interés.

Fuente de financiamiento: autofinanciado. 
Tabla 1. Contrapuntos y propuestas a argumentos comúnmente utilizados para usar hidroxicloroquina e ivermectina en la COVID-19.

\begin{tabular}{|c|c|c|}
\hline $\begin{array}{l}\text { Argumento } \\
\text { comúnmente } \\
\text { utilizado }\end{array}$ & Contrapunto & Propuestas \\
\hline $\begin{array}{l}\text { «La } \\
\text { hidroxicloroquina } \\
\text { funciona. Así } \\
\text { lo demuestra } \\
\text { la experiencia } \\
\text { clínica» }\end{array}$ & $\begin{array}{l}\text { - No existe ninguna evidencia confiable de que la hidroxicloroquina es beneficiosa } \\
\text { para el tratamiento de la COVID-19. De hecho, hay evidencia confiable de que no } \\
\text { funciona. } \\
\text { - La experiencia clínica constituye el grado más bajo de evidencia sobre la eficacia } \\
\text { y seguridad de los medicamentos, pues los médicos son muy propensos a } \\
\text { cometer errores en atribuir si la mejoría de un paciente es debida a la medicina } \\
\text { o a la historia natural de la enfermedad en ese paciente. Esto es particularmente } \\
\text { cierto en enfermedades de presentación y curso tan variable como la COVID-19. } \\
\text { - Los ensayos clínicos aleatorios, por otro lado, constituyen el grado más alto/ } \\
\text { confiable de evidencia sobre la eficacia y seguridad de los medicamentos. } \\
\text { - Muy recientemente se han reportado } 2 \text { ensayos clínicos aleatorios importantes } \\
\text { con hidroxicloroquina en COVID-19. Ambos hallaron resultados negativos, es } \\
\text { decir, que la hidroxicloroquina no trajo beneficio ni en el tratamiento ni en la } \\
\text { prevención de la COVID-19, pero sí trajo mayores efectos adversos. }\end{array}$ & $\begin{array}{l}\text { - Descontinuar la } \\
\text { recomendación de } \\
\text { tratar la COVID-19 con } \\
\text { hidroxicloroquina. } \\
\text { - Evitar connotaciones } \\
\text { negativas sobre las } \\
\text { retracciones o cambios de } \\
\text { opinión de los expertos, que } \\
\text { ocurren a medida que sale } \\
\text { nueva evidencia y se tiene } \\
\text { un mejor conocimiento de la } \\
\text { enfermedad. } \\
\text { - Reevaluar críticamente y en }\end{array}$ \\
\hline $\begin{array}{l}\text { "La FDA aprobó } \\
\text { el uso de } \\
\text { ivermectina» }\end{array}$ & $\begin{array}{l}\text { - Si bien es cierto que la FDA ha aprobado hace mucho tiempo la ivermectina para } \\
\text { el tratamiento de parásitos intestinales, la FDA nunca ha aprobado el uso de } \\
\text { ivermectina para COVID-19. } \\
\text { - El artículo in vitro que evaluó la ivermectina en un plato de cultivo con células }{ }^{[10]} \\
\text { proviene de Australia, no de la FDA, pero mencionó que este es un medicamento } \\
\text { aprobado por la FDA en su título, causando mucha confusión al respecto. } \\
\text { - La FDA también ha prohibido la utilización de preparaciones para animales de } \\
\text { este medicamento. }\end{array}$ & $\begin{array}{l}\text { forma periódica la evidencia } \\
\text { sobre ensayos clínicos } \\
\text { aleatorios bien diseñados. }\end{array}$ \\
\hline $\begin{array}{l}\text { "La ivermectina } \\
\text { funciona» }\end{array}$ & $\begin{array}{l}\text { - No existe evidencia confiable de que la ivermectina funciona para el tratamiento } \\
\text { de la COVID-19. } \\
\text { - La experiencia clínica constituye el grado más bajo de evidencia sobre la eficacia } \\
\text { y seguridad de los medicamentos, pues los médicos son muy propensos a } \\
\text { cometer errores en atribuir si la mejoría de un paciente es debida a la medicina } \\
\text { o a la historia natural de la enfermedad en ese paciente. Esto es particularmente } \\
\text { cierto en enfermedades de presentación y curso tan variable como el COVID-19. } \\
\text { - Los estudios que muestran un efecto antiviral de la ivermectina contra el } \\
\text { virus que produce la COVID-19 no fueron hechos en ningún ser vivo (animal o } \\
\text { humano) sino más bien en células aisladas de mono en un plato de cultivo [10]. } \\
\text { Sin embargo, las concentraciones necesarias para lograr un efecto antiviral en } \\
\text { estos experimentos fueron aproximadamente } 35 \text { a } 100 \text { veces mayores que las } \\
\text { que se alcanzan en humanos con las dosis recomendadas para uso médico. } \\
\text { - El estudio retrospectivo que sugirió que pacientes con COVID-19 tratados con } \\
\text { ivermectina tuvieron mejores resultados fue hecho con una base de datos no } \\
\text { confiable, que ha llevado a la retracción de este estudio incluso antes de que se } \\
\text { publicara en una revista médica. La dudosa veracidad y falta de confiabilidad de } \\
\text { esta base de datos llevó a retracciones similares de otros estudios de revistas } \\
\text { médicas importantes (The Lancet y New England Journal of Medicine). } \\
\text { - Los estudios retrospectivos no constituyen evidencia confiable sobre la eficacia y } \\
\text { seguridad de los medicamentos. } \\
\text { - No existe ningún ensayo clínico aleatorio con ivermectina en CovID-19. }\end{array}$ & $\begin{array}{l}\text { - Descontinuar la } \\
\text { recomendación de tratar } \\
\text { rutinariamente la COVID-19 } \\
\text { con ivermectina. } \\
\text { - Permitir su uso en el contexto } \\
\text { de estudios de investigación } \\
\text { debidamente justificados, } \\
\text { controlados, monitorizados y } \\
\text { regulados. } \\
\text { - Evitar connotaciones } \\
\text { negativas sobre las } \\
\text { retracciones o cambios de } \\
\text { opinión de los expertos, que } \\
\text { ocurren a medida que sale } \\
\text { nueva evidencia y se tiene } \\
\text { un mejor conocimiento de la } \\
\text { enfermedad. } \\
\text { - Reevaluar críticamente y en } \\
\text { forma periódica la evidencia } \\
\text { sobre ensayos clínicos } \\
\text { aleatorios bien diseñados. }\end{array}$ \\
\hline $\begin{array}{l}\text { «Es inhumano } \\
\text { no tratar con } \\
\text { nada», o «hay } \\
\text { que dar algo», o } \\
\text { "el paciente está } \\
\text { grave, se tiene que } \\
\text { hacer algo» }\end{array}$ & $\begin{array}{l}\text { - Uno de los pilares de la medicina es primum non nocere que significa «lo } \\
\text { primero es no hacer daño». } \\
\text { - Toda intervención médica puede provocar daño al paciente. Los actos médicos } \\
\text { hechos con las mejores intenciones pueden tener consecuencias indeseables, } \\
\text { incluso graves o letales. } \\
\text { - No se sabe si estos medicamentos son seguros en pacientes con COVID-19, que } \\
\text { tienen una fisiología orgánica muy diferente a los pacientes con artritis, lupus o } \\
\text { parasitosis (enfermedades en las que se han utilizado la hidroxicloroquina y la } \\
\text { ivermectina con pocos efectos adversos). }\end{array}$ & $\begin{array}{l}\text { - Aplicar solamente terapias } \\
\text { comprobadas (ver texto). } \\
\text { - Aplicar terapia de soporte } \\
\text { respiratorio y cuidados } \\
\text { intensivos de acuerdo con los } \\
\text { recursos existentes. } \\
\text { - Informar a la población sobre } \\
\text { los potenciales riesgos de los } \\
\text { tratamientos no comprobados. }\end{array}$ \\
\hline
\end{tabular}


Tabla 1. Contrapuntos y propuestas a argumentos comúnmente utilizados para usar hidroxicloroquina e ivermectina en la COVID-19 (Viene de la pág. anterior).

\section{Argumento comúnmente utilizado}

«Estos son medicamentos seguros y se han dado millones de dosis sin mayores efectos adversos»

«Si no funciona, no se pierde nada»

«Nadie discute

la necesidad de ensayos aleatorizados, pero van a tomar varios meses. Hasta ese entonces, ¿cuántos muertos habrá?»
- Un medicamento seguro o inocuo en un tipo de pacientes (como aquellos con artritis o lupus) podría resultar faltamente peligroso en otro tipo de pacientes (como aquellos con COVID-19).

- La hidroxicloroquina, por ejemplo, puede causar alteraciones del ritmo cardiaco que pueden ser mortales. Estas arritmias son muy raras en pacientes con lupus o artritis. Sin embargo, los pacientes con COVID-19 tienden a presentar diarrea, bajos niveles de potasio en sangre, e injuria del músculo del corazón, todo lo cual predispone a las mismas alteraciones del ritmo que causa la hidroxicloroquina. Estas arritmias son particularmente peligrosas en situaciones de bajos recursos en los que no se hace monitoreo cardiaco adecuado. La hidroxicloroquina puede provocar diarrea, que si bien es cierto se considera poco importante en pacientes con lupus o artritis, en pacientes con COVID-19 podría incrementar la transmisión viral por excreción del virus en las heces, aunque aún no está claro si el virus se transmite efectivamente por esta vía.

- La ivermectina es considerada segura, pero esta presunción está basada mayormente en la experiencia del uso de ivermectina en pacientes con parasitosis que generalmente son más jóvenes y no presentan otras enfermedades crónicas (de corazón, riñón, pulmón, hígado y sangre) como presentan muchos pacientes con COVID-19.

- Más aún, no conocen bien las interacciones medicamentosas entre la ivermectina y otros medicamentos usados en el tratamiento de la COVID-19, sobre todo en los pacientes con enfermedades crónicas y los críticamente enfermos. Por ejemplo, hay estudios que sugieren una interacción con la dexametasona que probablemente deba ser tratamiento estándar para COVID-19 (pendiendo la evaluación de los resultados completos del estudio RECOVERY).

- Podríamos estar haciendo daño a los pacientes dados los potenciales efectos adversos.

- Los pacientes que sí requieren estas medicinas para enfermedades en las que sí funcionan (como artritis o lupus), pueden quedar desabastecidos, lo que puede causar reagudización de su enfermedad, incluso con consecuencias graves.

- Un énfasis excesivo en estas medicinas disminuye la capacidad para aplicar o estudiar otras intervenciones de bajo costo que podrían ser beneficiosas, o incluso terapias que ya tienen beneficio comprobado.

- Existe un costo económico, que podría destinarse a intervenciones médicas o logísticas más útiles como: distanciamiento social, lavado de manos y uso de mascarillas.

- La pregunta formulada de esta manera podría transmitir involuntariamente un mensaje de que las medicinas en efecto reducen la mortalidad, lo que puede contribuir a la ansiedad de la población por conseguir y tomar los medicamentos, arriesgando contagios y toxicidad.

- La verdadera pregunta es: ¿Cuántos muertos habrá si se continúa recomendando agresivamente estos medicamentos sin evidencia de eficacia versus si se descontinúa su uso rutinario? No existen buenos motivos para suponer que el número sea menor con la primera estrategia, y de hecho podría ser mayor, a consecuencia de:

1. Toxicidad directa de los medicamentos.

2. Uso no regulado, incluyendo automedicación, sobredosis, y en el caso de la ivermectina, administración de preparaciones veterinarias.

3. Costo de oportunidad de enfatizar varias otras intervenciones importantes, como la educación, soporte psicológico y social del paciente, el manejo sintomático, el aislamiento, el rastreo de contactos, la monitorización cercana de la evolución clínica, el uso de medicamentos con eficacia establecida, etc.

4. Una excesiva confianza del paciente infectado que recibe el medicamento, lo que puede impedir la búsqueda de atención cuando hay deterioro sintomático, o puede reducir la alerta para evitar los contagios.

- Se podría promover a nivel de estado la ejecución rápida de ensayos a gran escala que den respuestas más prontas con evidencia de alta calidad.
Promover el enrolamiento de pacientes en ensayos clínicos de medicamentos prometedores, auspiciados por entidades públicas o privadas. Ejecutar rápidamente ensayos clínicos aleatorizados de gran escala, auspiciados por el gobierno en el que los pacientes se tratan con medicamentos prometedores de bajo costo, a medida que se recolectan datos.

El promover el uso de medicamentos en contextos donde no hay suficiente personal médico para brindar atención e información apropiada, puede motivar a la automedicación y el descuido de las medidas de prevención como el distanciamiento físico, el uso de mascarillas y el lavado de manos.

- Existen zonas remotas en el país, con población vulnerable, que se podrían beneficiar de drogas que disminuyan la mortalidad, si se impulsa el desarrollo de ensayos clínicos de manera urgente. 


\section{ORCID:}

Julio A. Chirinos: https://orcid.org/0000-0001-9035-5670 Víctor F. Corrales-Medina: https://orcid.org/0000-0002-9691-491X Gustavo Heresi-Dávila: https://orcid.org/0000-0002-9797-2599 Adrian V. Hernandez: http://orcid.org/0000-0002-9999-4003 Germán Málaga: https://orcid.org/0000-0002-7828-300X Jorge M. Mallea: https://orcid.org/0000-0003-4962-8838 J. Jaime Miranda: https://orcid.org/0000-0002-4738-5468 Oscar O. Morey: https://orcid.org/0000-0001-9599-5415 Juan E. Rodríguez Mori: https://orcid.org/0000-0002-9794-0327 Jesús E. Salinas-Gamero: https://orcid.org/0000-0001-9517-2427 José Serpa-Alvarez: https://orcid.org/0000-0001-8179-7290 Alvaro Taype-Rondan: https://orcid.org/0000-0001-8758-0463 Carol Zavaleta: https://orcid.org/0000-0003-4969-8959

\section{REFERENCIAS BIBLIOGRÁFICAS}

1. Ministerio de Salud del Perú. Prevención, Diagnóstico y Tratamiento de personas afectadas por COVID-19 en el Perú. Resolución Ministerial N 193-2020-MINSA [Internet]. Lima: MINSA; 13 de abril de 2020 [citado el 17 de junio de 2020]. Disponible en: https:// www.gob.pe/institucion/minsa/normas-legales/473575-193-2020minsa.

2. Ministerio de Salud del Perú. Prevención, Diagnóstico y Tratamiento de personas afectadas por COVID-19 en el Perú. Resolución Ministerial N 270-2020-MINSA [Internet]. Lima: MINSA; 8 de Mayo de 2020 [citado el 17 de junio de 2020]. Disponible en: https://www. gob.pe/institucion/minsa/normas-legales/563764-270-2020-minsa.

3. Hernandez AV, Roman YM, Pasupuleti V, Barboza JJ, White CM. Hydroxychloroquine or Chloroquine for Treatment or Prophylaxis of COVID-19: A Living Systematic Review. Ann Intern Med. 2020. doi: 10.7326/M20-2496.

4. Boulware DR, Pullen MF, Bangdiwala AS, Pastick KA, Lofgren SM, Okafor EC, et al. A Randomized Trial of Hydroxychloroquine as Postexposure Prophylaxis for Covid-19. N Engl J Med. 2020. doi: 10.1056/NEJMoa2016638.

5. Roden DM, Harrington RA, Poppas A, Russo AM. Considerations for Drug Interactions on QTC Interval in Exploratory COVID-19 Treatment. J Am Coll Cardiol. 2020;75(20):2623-2624.
6. Bonow RO, Hernandez AF, Turakhia M. Hydroxychloroquine, Coronavirus Disease 2019, and QT Prolongation. JAMA Cardiol. 2020. doi: 10.1001/jamacardio.2020.1782.

7. Juurlink DN. Safety considerations with chloroquine, hydroxychloroquine and azithromycin in the management of SARS-CoV-2 infection. CMAJ. 2020;192(17):E450-E453; doi: 10.1503/cmaj.200528.

8. Chorin E, Wadhwani L, Magnani S, Dai M, Shulman E, NadeauRouthier C, et al. QT interval prolongation and torsade de pointes in patients with COVID-19 treated with hydroxychloroquine/ azithromycin. Heart Rhythm. 2020;S1547-5271(20)30435-5. doi:10.1016/j.hrthm.2020.05.014.

9. Cepelowicz-Rajter J, Sherman M, Fatteh N, Vogel F, Sacks J, Rajter J-J. ICON (Ivermectin in COvid Nineteen) study: Use of Ivermectin is Associated with Lower Mortality in Hospitalized Patients with COVID19. medRxiv. 2020.06.06.20124461. doi: 10.1101/2020.06.06.20124461.

10. Caly L, Druce JD, Catton MG, Jans DA, Wagstaff KM. The FDAapproved drug ivermectin inhibits the replication of SARSCoV-2 in vitro. Antiviral Res. 2020;178:104787. doi: 10.1016/j. antiviral.2020.104787.

11. Horby P, Shen W, Emberson J, Mafham M, Bell J, Linsel, et al. RECOVERY Collaborative Group. Effect of Dexamethasone in Hospitalized Patients with COVID-19: Preliminary Report. MedRxiv. 2020.06.22.2013727. doi: 10.1101/2020.06.22.20137273

12. Goldman JD, Lye DCB, Hui DS, Marks KM, Bruno R, Montejano $R$, et al. Remdesivir for 5 or 10 Days in Patients with Severe Covid-19. N Engl J Med. 2020. doi: 10.1056/NEJMoa2015301.

13. Beigel JH, Tomashek KM, Dodd LE, Mehta AK, Zingman BS, Kalil AC. Remdesivir for the Treatment of Covid-19 - Preliminary Report. N Eng J Med. 2020. doi: 10.1056/NEJMoa2007764.

14. Wang $Y$, Zhang $D$, Du G, Du R, Zhao J, Jin $Y$, et al. Remdesivir in adults with severe COVID-19: a randomised, double-blind, placebo-controlled, multicentre trial. Lancet. 2020;395:1569-78. doi: 10.1016/\$0140-6736(20)31022-9.

15. Gilead press release: Gilead Announces Results From Phase 3 Trial of Remdesivir in Patients With Moderate COVID-19 [Internet]. Gilead; 2020 [citado el 17 de junio de 2020]. Disponible en: https://www.gilead.com/news-and-press/press$\mathrm{room} /$ press-releases/2020/6/gilead-announces-results-fromphase-3-trial-of-remdesivir-in-patients-with-moderate-covid-19. 